\title{
Mode locking by a frequency-doubling crystal: generation of transform-limited ultrashort light pulses
}

\author{
Krassimir Stankov \\ Max-Planck-Institut für biophysikalische Chemie, Abteilung Laserphysik, Am Fassberg, D-3400 Göttingen, Federal Republic of Germany
}

Received September 28, 1988; accepted January 12, 1989

\begin{abstract}
Transform-limited ultrashort green and infrared light pulses have been generated from a Nd:YAG laser mode locked by an intracavity second-harmonic crystal in combination with a dichroic output coupler. The mode-locking process is based on substantial reconversion of the second-harmonic radiation back into fundamental radiation after reflection by the dichroic mirror. With this technique light pulses as short as $45 \mathrm{psec}$ have been generated.
\end{abstract}

In a preliminary experiment ${ }^{1,2}$ a new method of passive mode locking that uses intracavity second-harmonic generation was demonstrated. The method is based on nonlinear reflection achieved by the combination of a frequency doubler and a dichroic mirror. In contrast to the common case of intracavity secondharmonic generation, the output laser mirror is a total reflector at the second-harmonic wavelength. The nonlinear reflection is based on generation of the second harmonic in the crystal and amplification of the fundamental radiation after reflection by the dichroic mirror owing to the presence of the totally reflected second harmonic. To reverse the interaction between the reflected waves (fundamental and second harmonic) a proper phase relationship is provided. As a result the reflectivity of the crystal-mirror combination (referred to as a nonlinear mirror) increases when the light intensity increases. ${ }^{3}$

This nonlinear device with properties similar to those of the saturable absorbers was used to mode lock a pulsed Nd:YAG laser. In preliminary experiments 100-psec pulses with a pronounced substructure were generated. This Letter reports for the first time to my knowledge the generation of transform-limited ultrashort light pulses with this new technique and points out the limitations when type II (oe-e) phase matching is used for frequency doubling.

The experimental setup, depicted in Fig. 1, is similar to the original one. ${ }^{2}$ The cavity length was increased to $2 \mathrm{~m}$ for future experiments with active mode locking and to insert a harmonic splitter (total reflector at $1.06 \mu \mathrm{m}$, fully transmitting at $0.53 \mu \mathrm{m}$ ) between the telescope and the second-harmonic-generation crystal. The antireflection-coated telescope (magnification $3.75 \times$ ) provides $\mathrm{TEM}_{00}$ operation with a beam diameter of $1.2 \mathrm{~mm}$ at the nonlinear crystal. A polarizer was placed between the laser rod and the cavity end mirror to provide a definite polarization of the laser radiation and easy orientation of the frequency doubler. The uncoated KTP frequency doubler was replaced by a 6-mm-long crystal of the same type but with antireflection coatings (Airtron-Litton). The entrance side of the crystal is antireflection coat- ed for the fundamental and faces the laser rod, while the exit side is antireflection coated for the second harmonic and faces the dichroic mirror.

These modifications were aimed at facilitating the generation of shorter and structure-free pulses. Indeed the extraction of the second harmonic from the laser cavity after the second phase through the crystal prevents the reinjection of a second-harmonic wave with a wrong phase back into the nonlinear crystal. Although it is strongly attenuated by the laser rod, this reinjected radiation may cause the appearance of the observed substructure. The nonlinear crystal with highly parallel faces used in the preliminary experiments was situated almost normally to the cavity axes and acted as a Fabry-Perot spectral selector, thus limiting the minimum pulse duration and possibly giving rise to the pulse structure as well.

The laser was operated at a $1-2-\mathrm{Hz}$ repetition rate. Careful adjustment of the crystal-mirror separation ${ }^{2}$ and the pumping rate resulted in generation of typical mode-locked pulse trains of 400-nsec (FWHM) duration. The probability of generating satellite-free pulse trains was approximately $80 \%$. Deviation from the optimum pumping rate to lower or higher levels deteriorated the mode locking and resulted in the appearance of satellites. The output energy at the fun-



Fig. 1. Experimental setup for generating transform-limited ultrashort laser pulses by frequency-doubling mode locking. M1, total reflector mirror; $\mathrm{P}$, polarizer; $\mathrm{T}$, telescope; HS, harmonic splitter; KTP, frequency-doubling crystal; $\mathrm{DM}$, dichroic mirror (total reflector at $532 \mathrm{~nm}, 25 \%$ reflection at $1064 \mathrm{~nm}$ ). 


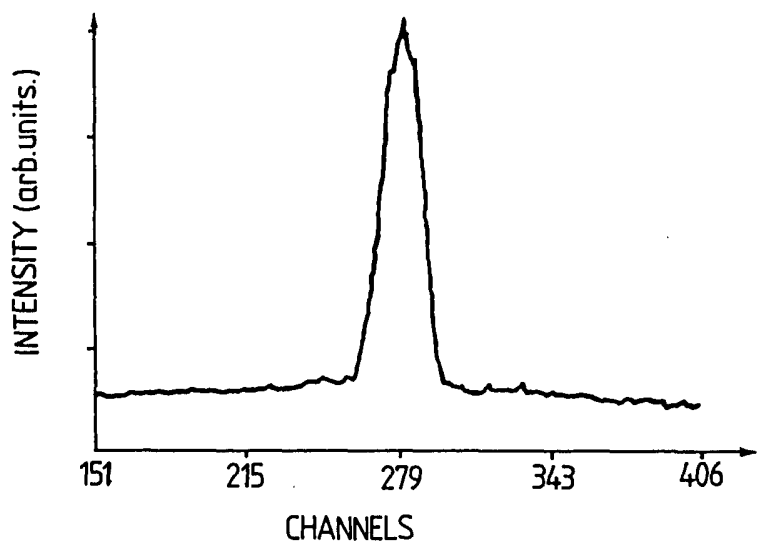

Fig. 2. Streak-camera record of an individual ultrashort laser pulse at $532 \mathrm{~nm}$ revealing a pulse duration of $42 \mathrm{psec}$ (FWHM). The sweep rate is $2.36 \mathrm{psec} / \mathrm{ch}$ annel.

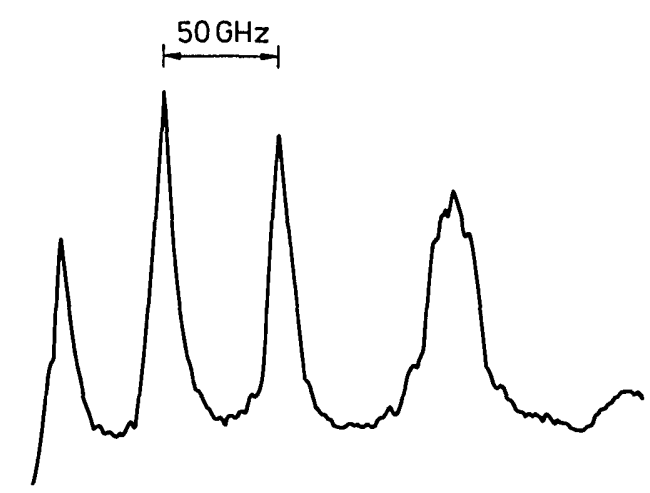

Fig. 3. Fabry-Perot pattern of the laser radiation. The free spectral range is $50 \mathrm{GHz}$.

damental wavelength available at the dichroic output coupler was typically $8 \mathrm{~mJ}$, while the second-harmonic output emerging from the harmonic splitter was 0.8 mJ. If we take into account that after reflection by the output coupler partial reconversion back into fundamental takes place, the actual conversion efficiency for a single,pass in the direction from the laser rod to the dichroic mirror was calculated to exceed $20 \%$. When the laser failed to mode lock the output energy was lower by approximately $40 \%$, as observed earlier. ${ }^{2}$

The individual pulse parameters were measured at the second-harmonic output. Pulse-width measurements were performed using a streak camera (Hamamatsu Temporal Disperser C 1587). The disappearance of satellites and a gradual decrease in the pulse duration were observed toward the end of the pulse train. Smooth and structure-free pulses having a duration between 40 and 50 psec were measured in the second half of the train (Fig. 2).

The failure to generate shorter pulses is probably due to the limited number of round trips. Indeed it has been shown ${ }^{4}$ that the nonlinear mirror has somewhat lower pulse-shortening capabilities compared with the saturable absorbers. Thus a longer pulse evolution may be needed to obtain shorter pulses. In pulsed lasers this may be achieved by using feedback control, ${ }^{5}$ which extends the pulse-train duration.

We have investigated the spectral content of the laser generation using a Fabry-Perot interferometer (Tropel Model 350) with 50- and 100-GHz free spectral ranges. The Fabry-Perot pattern was analyzed by a linear diode-array system (Dioscope, Sears) and observed on a digital storage oscilloscope. These measurements revealed a spectral width of $10 \mathrm{GHz}$ (Fig. 3). If a Gaussian pulse shape is assumed, transform-limited pulses are generated in the second half of the pulse train. Here we note that the measured spectrum has been integrated along the whole pulse train, and at the beginning of the pulse train the spectrum is narrower. According to the frequency-domain description of the second-harmonic mode-locking process, ${ }^{6}$ a reflection by the nonlinear mirror broadens the spectrum. When the laser radiation is passing through the crystal in the direction from the laser rod to the dichroic mirror the generated second-harmonic field is composed of mixed terms originating from the various longitudinal modes. After reflection by the dichroic mirror the difference-frequency generation between the second-harmonic radiation and the various longitudinal modes of the fundamental radiation produces sideband frequencies, thus broadening the spectrum. The spectral broadening will terminate at a certain point owing to the combined action of the active-medium gain profile and the eventual pressence of an intracavity spectral limiter. In our case the oe-e type of KTP crystal may act as a spectral selector, thus limiting the minimum pulse duration. In this experiment the 6-mm-long crystal and the polarizer form a birefringent filter with a 1.1-nm $(290-\mathrm{GHz})$ free spectral range. This gives a bandwidth of approximately half of the free spectral range, which is comparable with the Nd:YAG fluorescence width of $120 \mathrm{GHz}$. In this respect it should be pointed out that the oe-e type of frequency doublers are not suitable for generating extremely short pulses from lasers with larger spectral widths, e.g., Nd:glass lasers. Use of the oo-e type of crystal would avoid this problem, since it does not act as a birefringent filter. Then the only limitation arising from the nonlinear mirror would be the groupvelocity dispersion in the nonlinear crystal, as discussed earlier. ${ }^{2,3}$

In conclusion, we have demonstrated generation of transform-limited light pulses from a Nd:YAG laser mode locked by a second-harmonic nonlinear crystal. The minimum pulse duration of approximately 45 psec is probably due to the limited time of the pulse evolution.

The author acknowledges stimulating discussions with and the support of F.P. Schäfer, the help of J. Jethwa in the experiment, and a research fellowship from the Alexander von Humboldt Foundation and thanks the referees for their valuable comments.

The author is on leave from the Department of Physics, Sofia University, BG-1126 Sofia, Bulgaria. 


\section{References}

1. K. A. Stankov, in Digest of Conference on Lasers and Electro-Optics (Optical Society of America, Washington, D.C., 1988), paper ThJ4.

2. K. A. Stankov and J. Jethwa, Opt. Commun. 66, 41 (1988).
3. K. A. Stankov, Appl. Phys. B 45, 191 (1988).

4. K. A. Stankov" "Pulse shortening by a nonlinear-mirror mode-locker," Appl. Opt. (to be published).

5. P. Heinz, W. Kriegleder, and A. Laubereau, Appl. Phys. A 43, 209 (1987).

6. K. A. Stankov, "The passive mode-locking using intracavity frequency doubling" (unpublished observations). 